\title{
Neutron Diffraction Studies of Nanoparticle $\mathrm{DyMnO}_{3}$ Compound
}

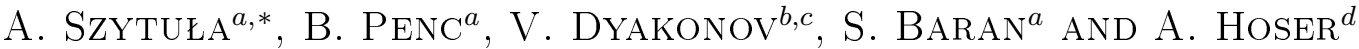 \\ ${ }^{a}$ M. Smoluchowski Institute of Physics, Jagiellonian University, W.S. Reymonta 4, 30-059 Kraków, Poland \\ ${ }^{b}$ Institute of Physics, PAS, al. Lotników 32/46, 02-668 Warszawa, Poland \\ ${ }^{c}$ A.A. Gałkin Donetsk Physico-Technical Institute, NANU, R. Luxemburg 72, 83114 Donetsk, Ukraine \\ ${ }^{d}$ Helmholtz-Zentrum Berlin, Hahn-Meitner Pl. 1, 14109 Berlin, Germany
}

(Received May 20, 2013; in final form November 5, 2013)

The neutron powder diffraction (NPD) measurements of the nano-size $\mathrm{DyMnO}_{3}$ manganite have been performed. The obtained results indicate that this compound crystallizes in the orthorhombic crystal structure described by the space group Pnma. The Mn and Dy moments order antiferromagnetically at different temperatures and form modulated magnetic structure described by the propagation vector $\boldsymbol{k}=\left(k_{x}, 0,0\right)$ with the different values of $k_{x}$ component for the $\mathrm{Mn}$ and Dy sublattices. The values of $k_{x}$ component for Mn sublattice increase with decreasing of the temperature and are smaller that in bulk compound. The wide Bragg peaks related to the Dy sublattice suggest that the magnetic order in this sublattice has the cluster-like character.

DOI: $10.12693 /$ APhysPolA.125.65

PACS 61.05.F-, 75.25.-j, 75.50.Ee

\section{Introduction}

The family of perovskite-like oxides $\mathrm{RMnO}_{3}$, where $\mathrm{R}$ are rare earth elements, has been extensively studied in recent years. These investigations concentrate also on the influence of grain size on the magnetic properties. The neutron diffraction data for $\mathrm{DyMnO}_{3}$ compounds indicates the change of the parameters of the magnetic ordering with the change of the grain size [1].

This work reports the results of the neutron diffraction measurements for the isostructural $\mathrm{DyMnO}_{3}$ nanoparticle compound. At room temperature, the bulk $\mathrm{DyMnO}_{3}$ compound exhibits the orthorhombically distorted perovskite structure (space group Pnma). The magnetic moments in particularly sublattices are order in different temperatures: at $39 \mathrm{~K}$ for $\mathrm{Mn}$ and $6.5 \mathrm{~K}$ for Dy sublattices. At low temperatures the magnetic moments in both sublattices form sine-modulated structure described by the propagation vector $\boldsymbol{k}=\left(k_{x}, 0,0\right)$ with $k_{x}$ equal to 0.405 for Mn site and 0.5 for Dy site [2].

Investigated in this work sample of nanosize $\mathrm{DyMnO}_{3}$ manganite is prepared by the sol-gel method described in Ref. [3]. The X-ray data at room temperature indicate that the sample has orthorhombic structure. The grain size was determined using the Scherrer relation $d=(\lambda / B) \cos \theta_{\mathrm{B}}$, where $d$ is the grain size, $\lambda=1.5418 \AA$ - the X-ray wavelength, $\theta_{\mathrm{B}}$ - the corresponding angle of the Bragg diffraction and $B$ the difference between half-widths of the Bragg reflex of the nanopowder and the standard sample $\mathrm{Si}$ with the size grain equal to $10 \mu \mathrm{m}$. For the sample annealed at $850^{\circ} \mathrm{C}$ the average size grain is $48 \mathrm{~nm}$. The magnetic data indicate the Néel temperature for Dy sublattice equal to $8.3 \mathrm{~K}$. In this work

\footnotetext{
*corresponding author; e-mail: andrzej.szytula@uj.edu.pl
}

in purpose to explain microscopic properties concerning the crystal and magnetic structure of nanoparticle sample $\mathrm{DyMnO}_{3}$ the neutron diffraction experiment is reported.

\section{Experimental details and results}

The neutron diffraction experiment has been performed on the sample which was obtained by the sol-gel method and next annealed at $850^{\circ} \mathrm{C}[3]$. The neutron diffractograms were obtained at temperatures equal to $1.6,12,25$, and $50 \mathrm{~K}$ with the use of the $\mathrm{E} 6$ diffractometer at BERII reactor (Helmholtz-Zentrum Berlin). The incident neutron wavelength was $2.44 \AA$. The data were analyzed using the Rietveld-type program FullProf [4].

TABLE

Crystal structure parameters of nanosized $\mathrm{DyMnO}_{3}$ compound refined on the basis of the neutron diffraction patterns collected at 1.5 and $50 \mathrm{~K}$. Standard deviations are given in parentheses.

\begin{tabular}{c|c|c}
\hline \hline Parameters $/ T[\mathrm{~K}]$ & 1.5 & 50 \\
\hline$a[\AA]$ & $5.8265(8)$ & $5.8267(9)$ \\
$b[\AA]$ & $7.3621(12)$ & $7.3661(13)$ \\
$c[\AA]$ & $5.2762(8)$ & $5.2793(9)$ \\
$V\left[\AA^{3}\right]$ & $226.32(11)$ & $226.59(11)$ \\
$x_{\mathrm{Dy}}$ & $0.0833(7)$ & $0.0845(8)$ \\
$z_{\mathrm{Dy}}$ & $0.9846(9)$ & $0.9823(11)$ \\
$x_{\mathrm{O} 1}$ & $0.4652(15)$ & $0.4611(20)$ \\
$z_{\mathrm{O} 1}$ & $0.1122(18)$ & $0.1164(22)$ \\
$x_{\mathrm{O} 2}$ & $0.3302(15)$ & $0.3342(17)$ \\
$y_{\mathrm{O} 2}$ & $0.0507(10)$ & $0.0527(11)$ \\
$z_{\mathrm{O} 2}$ & $0.7006(16)$ & $0.7043(20)$ \\
$R_{\text {Bragg }}[\%]$ & 4.40 & 4.62 \\
$R_{\text {prof. }}[\%]$ & 3.57 & 3.55
\end{tabular}


The neutron diffraction patterns measured at different temperatures are shown in Fig. 1. The data confirm that investigated compound crystallizes in the orthorhombic crystal structure (space group Pnma) with the following distribution of atoms: the Dy and O1 atoms occupy the 4 (c) site: $x, 1 / 4, z$; the Mn atom 4 (b) site: $0,0,1 / 2$ while $\mathrm{O} 2$ atoms in the $8(\mathrm{~d})$ one: $x, y, z$. The crystal structure parameters determined from the data at 1.5 and $50 \mathrm{~K}$ are listed in Table. The analysis of these data indicates that the orthorhombic crystal structure is stable down to low temperatures.

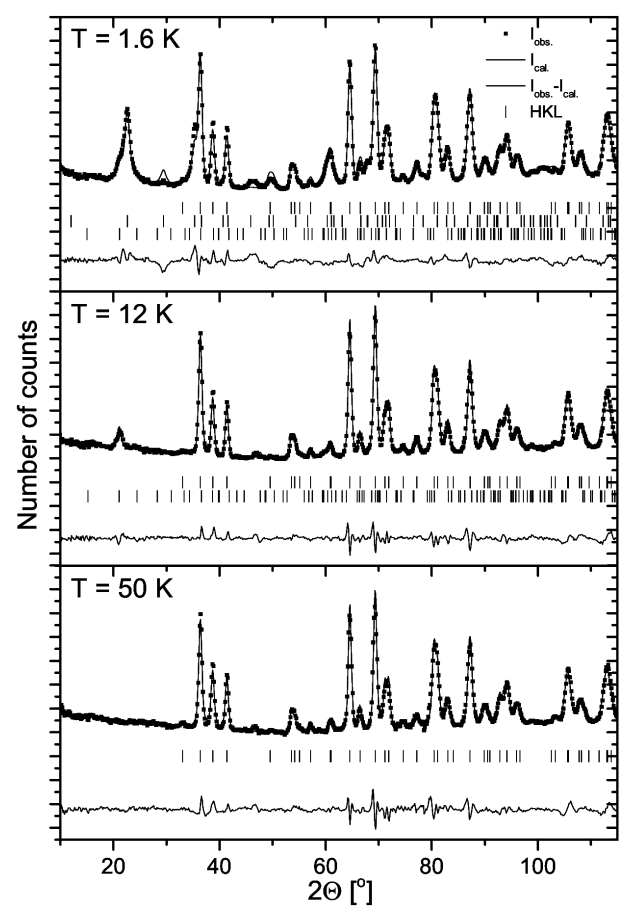

Fig. 1. Comparison between the observed and calculated NPD patterns by the Rietveld method at (a) $1.6 \mathrm{~K}$, (b) $12 \mathrm{~K}$, and (c) $50 \mathrm{~K}$. The symbols represent the experimental data while the solid line denotes the calculated profile. The difference between the observed and calculated intensities is shown at the bottom of each diagram. The vertical bars indicate the positions of the Bragg peaks, first row nuclear, second and third magnetic connected with the Mn- and Dy-sublattices.

On the neutron diffraction patterns at $1.6,12$, and $25 \mathrm{~K}$ the additional peaks connected with the magnetic origin are observed. These peaks can be indexed by propagation vector $\boldsymbol{k}=\left(k_{x}, 0,0\right)$. The values of $k_{x}$ components which described the ordering in $\mathrm{Mn}$ site are equal to $0.360(4)$, $0.370(2)$, and $0.376(4)$ at 25,12 , and $1.6 \mathrm{~K}$, respectively.

In crystal unit cell the $\mathrm{Mn}^{3+}$ ions in the $4(\mathrm{~b})$ site can be described by four Bravais lattices labeled as: Mn1 $(0,0,1 / 2), \operatorname{Mn} 2(1 / 2,0,0), \operatorname{Mn} 3(0,1 / 2,1 / 2)$, and Mn4 $(1 / 2,1 / 2,0)$ where $\mathrm{Dy}^{3+}$ ions in $4(\mathrm{c})$ : Dy1 $(x, 1 / 4, z)$, Dy2 $(1 / 2-x, 3 / 4,1 / 2+z)$, Dy $3(\bar{x}, 3 / 4, \bar{z})$ and Dy 4 $(1 / 2+x, 1 / 4,1 / 2-z)$. The analysis based on the Bertaut method [5] provides four modes: one ferromagnetic: $\boldsymbol{F}=$ $m_{1}+m_{2}+m_{3}+m_{4}$ and three antiferromagnetic ones:
$\boldsymbol{A}=m_{1}-m_{2}-m_{3}+m_{4}, \boldsymbol{C}=m_{1}+m_{2}-m_{3}-m_{4}$, and $\boldsymbol{G}=m_{1}-m_{2}+m_{3}-m_{4}$. Numerical analyses of the intensities of magnetic peak indicate that the magnetic ordering in the Mn sublattice is described by the $A_{x}$ mode. This magnetic order is stable up to $1.6 \mathrm{~K}$. The Mn-moments are parallel to the $a$-axis and form a collinear incommensurate structure with the crystal structure one. The magnetic moments localized on the Mn atoms are equal to $3.24(14), 3.74(12)$ and $4.24(11) \mu_{\mathrm{B}}$ at 25.12 and $1.6 \mathrm{~K}$, respectively. The magnetic $R$-factors are equal to $11.7,9.9$, and $10.1 \%$, respectively.

At $T=1.6 \mathrm{~K}$ the additional peaks corresponding to the magnetic ordering in Dy sublattice described by the $A_{x}$-mode are observed. The magnetic order is described by the propagation vector $\boldsymbol{k}=(0.501(12), 0,0)$. The Dy moments equal to $8.71(18) \mu_{\mathrm{B}}$ are parallel to the $a$-axis $\left(R_{\mathrm{mag}}=10.0 \%\right)$.

\section{Conclusions}

Presented in the work data indicate that the nanoparticle sample of $\mathrm{DyMnO}_{3}$ crystallizes in the orthorhombic crystal structure described by the space group Pnma similar to those for bulk material. The values of the structural parameters indicate the small influence of the grain size on atomic ordering.

Comparison of the data for nano and bulk samples suggest that the ordering in the Mn and Dy sublattices described by the propagation vector $\boldsymbol{k}=\left(k_{x}, 0,0\right)$ is similar. For a nanosample the values of the $k_{x}$ components for the Mn sublattice are smaller that for bulk material and indicate the incommensurate structure while for Dy sublattice similar as in bulk compound and corresponding to the commensurate one. The wide Bragg peaks related to the Dy sublattice suggests that magnetic order has the cluster-like character. Similar effect is observed in the isostructural nano $\mathrm{DyMnO}_{3}$ compound [1]. The value of the $\mathrm{Mn}$ magnetic moment is near the value for the $\mathrm{Mn}^{3+}$ ions $\left(4.0 \mu_{\mathrm{B}}\right.$ corresponding to $\left.S=2\right)$ while this for Dy moments is smaller that for $\mathrm{Dy}^{3+}$ ion $\left(10.0 \mu_{\mathrm{B}}\right)$ which suggests the influence of the crystal electric field.

\section{Acknowledgments}

This research project has been supported by the European Commission under the 7th Framework Programme through the "Research Infrastructure" action of the "Capacities" Programme, NM13-II grant No. 283883.

\section{References}

[1] W. Bażela, M. Dul, V. Dyakonov, Ł. Gondek, A. Hoser, J.-U. Hoffmann, B. Penc, A. Szytuła, Z. Kravchenko, I. Nosalev, A. Zarzycki, Acta Phys. Pol. A 121, 785 (2012).

[2] R. Feyerherm, E. Dudzik, N. Aliouane, D.N. Argyriou, Phys. Rev. B 73, R180401 (2006).

[3] V. Dyakonov, A. Szytuła, S. Baran, Z. Kravchenko, E. Zubov, O. Iessenchuk, W. Bażela, M. Dul, A. Zarzycki, H. Szymczak, Acta Phys. Pol. A 117, 607 (2010).

[4] J. Rodriguez-Carvajal, Physica B 192, 55 (1993).

[5] E.F. Bertaut, in: Magnetism, Vol. III, Eds. G.T. Rado, H. Shul, Academic Press, New York 1963, p. 149. 